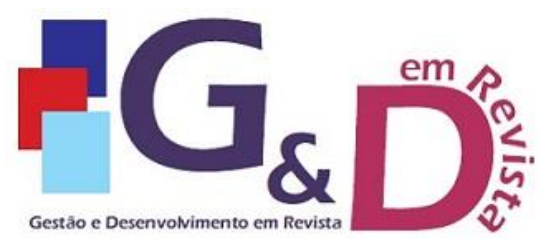

Gestão e Desenvolvimento em Revista

V. 7, N. 1, jan-jun/2021, p. 71-81.

ISSN online: $2446-8738$

Artigo recebido em: 27/05/2021

Artigo aprovado em: 16/08/2021

\title{
PRÁTICAS SUSTENTÁVEIS E GESTÃO AMBIENTAL NAS INSTITUIÇÕES DE ENSINO SUPERIOR: UM ESTUDO BIBLIOMÉTRICO NA WEB OF SCIENCE
}

\author{
Greice Eccel Pontelli \\ Mestre em Gestão de Organizações Públicas pela Universidade Federal de Santa Maria - UFSM. \\ Atualmente é Doutoranda no Programa de Pós-Graduação em Administração na Universidade \\ Federal de Santa Maria - UFSM. \\ E-mail: grace_pontelli@yahoo.com.br \\ karine da Silveira Aquino \\ Graduanda em Engenharia de Produção pela Universidade Federal de Santa Maria - UFSM. \\ E-mail: karine-aquino@hotmail.com

\section{Jordana Marques Kneipp} \\ Doutorado em Administração pelo Programa de Pós-graduação em Administração da Universidade \\ Federal de Santa Maria (PPGA/UFSM). Atualmente é Professora Adjunta do Departamento de \\ Ciências Administrativas da Universidade Federal de Santa Maria. \\ E-mail: jordana.kneipp@ufsm.br
}

\section{Resumo}

O estudo tem por objetivo apresentar um panorama da produção científica sobre práticas sustentáveis e gestão ambiental em Instituições de Ensino Superior na base de dados Web of Science (2010 a 2019) e identificar os Hot Topics. Os resultados indicam que as áreas temáticas com maior concentração de publicações são a Pesquisa Educacional, as Ciências Ambientais e a Tecnologia e Ciências Sustentáveis Verdes. Entre 2017-2019, o aumento nas pesquisas deixa em evidência a preocupação em investigações na temática. As áreas de Gestão, Estudos Ambientais, Ciências Ambientais, Negócios, Pesquisa Educacional, Engenharia Ambiental e Enfermagem são consideradas Hot Topics. Portanto, o conhecimento científico sobre sustentabilidade e gestão ambiental nas universidades pode ser aprofundado tanto a partir de uma perspectiva de gestão (práticas de gestão e governança) quanto acadêmica (educação ambiental e evolução das pesquisas).

Palavras-chave: Sustentabilidade; Universidades; Bibliometria.

\begin{abstract}
The study aims to present an overview of scientific production on sustainable practices and environmental management in Higher Education Institutions in the Web of Science database (from 2010 to 2019) and identify the Hot Topics. The results indicate that the thematic areas with the highest concentration of publications are: Educational Research, Environmental Sciences, and Technology and Green Sustainable Sciences. Between 2017-2019, the increase in research highlights the concern in investigations on the subject. Management, Environmental Studies, Environmental Sciences, Business, Educational Research, Environmental Engineering, and Nursing are considered Hot Topics. Therefore, scientific knowledge about sustainability and environmental management in universities can be deepened from a management perspective (management and governance practices) and academic (environmental education and research evolution).
\end{abstract}

Keywords: Sustainability; Universities; Bibliometrics. 


\section{INTRODUÇÃO}

Os problemas ambientais resultantes do acelerado processo de industrialização, do crescimento populacional e do aumento dos níveis de consumo dos recursos repercutiram na tomada de consciência da sociedade (SILVA; AZEVEDO, 2019). Nesse sentido, os princípios do Desenvolvimento Sustentável representam pontos-chave no desenvolvimento de políticas e de atividades das Instituições de Ensino Superior (IES), tanto pelo seu impacto no meio ambiente quanto pelo papel que desempenham na sociedade (RAGAZZI; GHIDINI, 2017). Espera-se que as Instituições de Ensino Superior se engajem na sustentabilidade tanto internamente (dentro da organização) quanto externamente, tornando-se um agente na comunidade (DAGILIŪTE்; LIOBIKIENE்; MINELGAITE், 2018).

Conforme Lozano et al. (2013), a maioria das IES ainda está atrasada em relação às empresas. Os gestores precisam se tornar mais proativos ao integrar o Desenvolvimento Sustentável como parte do sistema da organização, descartando velhos paradigmas (LOZANO et al., 2013). Os investimentos necessários para a sustentabilidade nas universidades são vistos como uma barreira, enquanto os benefícios a partir do desempenho ambiental e econômico são negligenciados (ÀVILA et al., 2017).

Devido ao alto impacto social, as IES são desafiadas a assumir um papel de liderança nas questões de sustentabilidade (DISTERHEFT et al., 2012). A inserção de políticas de Desenvolvimento Sustentável se trata de um mecanismo valioso para evidenciar o compromisso da IES com a sustentabilidade e para auxiliar na implementação de treinamento em sustentabilidade (LEAL FILHO et al., 2018). É importante que as organizações social, ambiental e economicamente corretas apliquem seus recursos para estimular a criação de uma sociedade sustentável (CAIADO et al., 2018).

Nesse sentido, o estudo tem por objetivo apresentar um panorama da produção científica sobre práticas sustentáveis e gestão ambiental em Instituições de Ensino Superior na base de dados Web of Science, no período de 2010 a 2019. Para tanto, realizou-se um estudo bibliométrico a fim de investigar as características da produção científica em determinada área. 
Destaca-se a importância desse estudo a fim de contribuir com 0 conhecimento científico, visto que pesquisadores poderão identificar novos insights para estudos futuros. O incentivo para pesquisas que abordam a temática da sustentabilidade em universidades é essencial para a ampliação da conscientização da sociedade e da comunidade acadêmica, uma vez que se trata do engajamento de organizações públicas e privadas em busca da mudança comportamental e cultural. Outrossim, acrescenta-se o papel de destaque das IES no campo do saber, as quais podem servir de modelo para outras organizações e disseminar suas práticas.

O presente estudo está estruturado em cinco seções. Após a introdução, a segunda seção apresenta o referencial teórico acerca da Sustentabilidade e Práticas Sustentáveis nas IES. Na seção 3, aborda-se o método. Em seguida, a seção 4 apresenta os resultados. Por fim, as considerações finais e as referências.

\section{REFERENCIAL TEÓRICO}

\subsection{SUSTENTABILIDADE}

No último século, as atividades humanas modificaram drasticamente os processos naturais ao mesmo tempo em que afetaram, significativamente, os sistemas socioecológicos, levando à atual crise ambiental (RUIZ-MALLÉN; HERAS, 2020). O desenvolvimento sustentável é aquele capaz de suprir as necessidades da geração atual sem comprometer a capacidade de atender as demandas das futuras gerações (BRUNDTLAND, 1991).

Por definição, o desenvolvimento sustentável procura diminuir diferenças sociais, prezar pelo meio ambiente e enfrentar limitações econômicas. Logo, é uma forma de identificar as necessidades da sociedade, de maneira que preserve e amplie recursos naturais e monetários (ARIVUMANI; VIJAYALAXMI, 2017). Ademais, com o Desenvolvimento Sustentável, busca-se alcançar a sustentabilidade (HOVE, 2004). Elkington (1997) introduziu o conceito do Triple Bottom Line, o qual integra as dimensões social, ambiental e econômica ao conceito de sustentabilidade.

Na perspectiva de Glaser (2012), o grande desafio do tema é a sua natureza interdisciplinar, conectando dimensões econômicas, ambientais e sociais. O autor usa como exemplo a mudança climática, que precisa ser analisada sob a ótica da 
segurança energética, dos alimentos e da água. Sendo assim, a interdisciplinaridade adiciona complexidade na mensuração e na criação de objetivos para o desenvolvimento sustentável.

As IES têm trabalhado formalmente em questões de sustentabilidade desde a Cúpula do Rio em 1992, por meio da implementação da Agenda 21; em 2002, a Organização das Nações Unidas (ONU) estabeleceu a Década das Nações Unidas de Educação para o Desenvolvimento Sustentável 2005-2014, que impulsionou a introdução de questões de sustentabilidade no currículo do ensino superior e nos sistemas de qualidade (RUIZ-MALLÉN; HERAS, 2020). Em 2014, na Conferência da UNESCO sobre Educação para o Desenvolvimento Sustentável, foi lançado o Programa de Ação Global da ONU, dando continuidade à Década da Educação para o Desenvolvimento Sustentável (RUIZ-MALLÉN; HERAS, 2020).

A sustentabilidade se tornou reconhecida e trata-se de uma das principais preocupações da atualidade, sendo que a atenção foi aumentada quando a Organização das Nações Unidas (ONU) estabeleceu a meta para atingir os 17 Objetivos do Desenvolvimento Sustentável até 2030 (UMAR, 2020). Nesse ínterim, a ONU criou a Agenda 2030, a qual indica 17 Objetivos para a Desenvolvimento Sustentável (ODS) para enfrentar os desafios globais. Os ODS são um apelo global à ação para acabar com a pobreza, proteger o meio ambiente e o clima e garantir que as pessoas, em todos os lugares, possam desfrutar de paz e de prosperidade (ONU, 2020). Entretanto, na perspectiva de Caiado et al. (2018), existem alguns pontos críticos para alcançar os ODS: investimentos em educação; liderança política e governança; compromisso global integrado e parcerias; soluções inovadoras; e indicadores agregados e confiáveis. Nessa senda, destaca-se o protagonismo das universidades na busca pelo desenvolvimento sustentável, uma vez que apresentam uma responsabilidade social especial no desenvolvimento da sociedade, na educação de futuros líderes e na proliferação da conscientização sobre sustentabilidade (AMARAL; MARTINS; GOUVEIA, 2015).

Outrossim, as Instituições de Ensino Superior desempenham um papel de destaque no desenvolvimento tecnológico, na preparação de estudantes e na disseminação de conhecimento, que pode e deve ser aproveitado para a criação de uma sociedade sustentável a partir da conscientização em todos os níveis da 
organização (TAUCHEN; BRANDLI, 2006). A seguir, discorre-se sobre as práticas sustentáveis nas Instituições de Ensino Superior.

\subsection{PRÁTICAS SUSTENTÁVEIS NAS IES}

As IES estão buscando mecanismos para incentivar a implementação de práticas sustentáveis e disseminar a conscientização ambiental para garantir a utilização de recursos naturais às futuras gerações. A discussão acerca dos problemas ambientais e a busca por ações focadas para a manutenção do meio ambiente são temas em destaque em âmbito nacional e internacional (LARA et al., 2015).

A partir de um estudo na Universidade de Saskatchewan, no Canadá, Sayed, Kamal e Asmuss (2013) identificaram cinco áreas críticas para melhorar a performance da sustentabilidade da universidade: educação, pesquisa, operações, governança e envolvimento da comunidade. Velasquez et al. (2006) descrevem uma estrutura composta por quatro fases em um processo estratégico, no qual são analisadas estratégias e práticas realizadas pelos atores-chave de iniciativas de sustentabilidade em IES. Conforme os autores, as fases são: (1) desenvolver a visão de sustentabilidade para a universidade; (2) a missão; (3) a comissão de sustentabilidade para a criação de políticas, metas e objetivos; e (4) as estratégias de sustentabilidade.

$\mathrm{Na}$ perspectiva de Thomasshow (2014), nove elementos integram um campus sustentável (divididos em três grandes categorias): infraestrutura (energia, materiais e alimentos); comunidade (governança, investimento e bem-estar); e aprendizagem (currículo, interpretação e estética). Tauchen e Brandli (2006) mencionam duas correntes de desenvolvimento sustentável nas universidades: (1) a questão educacional com a influência na qualificação de acadêmicos, de forma que inclua a busca pelas matérias ambientais; e (2) as iniciativas relacionadas à implementação de um sistema de gestão sustentável em sua estrutura organizacional, para servir de exemplo prático sobre sustentabilidade.

Vários fatores influenciam a implementação de práticas de sustentabilidade nas universidades: instituições públicas ou privadas; tamanho; liderança da organização em sustentabilidade; e orientação política (JORGE et al., 2015). Uma 
das dificuldades para a implementação do desenvolvimento sustentável nas instituições de ensino é o seu planejamento. Leal Filho et al. (2019) identificaram que a falta de entendimento acerca do tema gera pouca relevância e que nem todas as universidades possuem um planejamento estratégico para inserir o tema em sua comunidade acadêmica.

Leal Filho et al. (2018), no estudo realizado em universidades públicas e privadas de cinco países, destacam que a existência de políticas de Desenvolvimento Sustentável (DS) não é uma pré-condição para o engajamento no tema; todavia, as universidades que implementaram políticas de DS apresentaram maior probabilidade de inserir práticas como campus verde, desenvolvimento sustentável nos currículos e ações comunitárias. Nesse sentido, Rohrich e Takahashi (2019) realizaram um estudo bibliométrico em periódicos nacionais da área de sustentabilidade a partir de dois eixos: operacional e acadêmico. No eixo Operacional, estão a proposição e a aplicação de modelos, a gestão de resíduos em IES e as práticas de gestão ambiental. O eixo Acadêmico contempla a Educação ambiental em IES e a evolução das pesquisas.

A Agenda 21, assinada durante a ECO-92, a Agenda Ambiental da Administração Pública (A3P), instituída em 1999, o Plano de Logística Sustentável (PLS) e a Agenda 2030 são ferramentas que norteiam os gestores quanto à inserção de critérios de sustentabilidade. Do ponto de vista da gestão, Hall et al. (2014) apontam para a necessidade de ampliação de práticas sustentáveis e de gestão ambiental em processos de compras públicas. Portanto, a partir da literatura sobre 0 tema, percebe-se que, mesmo a comunidade acadêmica empenhada com questões ambientais, ainda se faz necessário a ampliação de iniciativas da gestão nas universidades. Leal Filho et al. (2019) destacam que uma comunicação mais efetiva facilita a participação de todos e a inserção do tema em seus projetos e pesquisas. Na próxima seção, apresenta-se o método do estudo.

\section{MÉTODO}

Este estudo foi desenvolvido a partir de uma pesquisa descritiva, com coleta de dados bibliográficos (ROSTAING, 1997) de natureza quantitativa (HAIR et., 2005). A bibliometria contribui com o conhecimento científico, visto que ajuda a 
conhecer o estágio em que a pesquisa em determinada área se encontra (MACEDO; CASA NOVA; ALMEIDA, 2010) e analisa a atividade científica por meio do estudo quantitativo das publicações (SILVA, 2004).

A fim de ampliar a discussão acerca das publicações sobre práticas sustentáveis e gestão ambiental nas IES/universidades, realizou-se uma busca na Web of Science compreendendo o período 2010-2019. Para tanto, utilizou-se os seguintes termos de busca: sustainable practices (práticas sustentáveis) ou environmental management (gestão ambiental) e universities (universidades) ou higher education institutions (instituições de ensino superior). Considerou-se o período de análise de 2010 a 2019. Destaca-se a escolha da base de dados da Web of Science porque abrange 12.000 periódicos e possui ferramentas que permitem a realização de estudos bibliométricos (CAPES, 2020).

A partir dos componentes disponíveis na Web of Science, foi possível identificar as características gerais das publicações e os 'índices h-b' e 'm'. Para tanto, utilizou-se as definições de Banks (2006) para análise dos índices:

- Índice h-b: Obtido pelo número de citações de um tópico ou pela combinação em determinando período, listados em ordem decrescente de citações.

- Índice m: Obtido pela divisão do índice h-b pelo período de anos que se deseja pesquisar.

As definições propostas por Banks (2006) são apresentadas no Quadro 1.

Quadro 1 - Definições para análise dos hot topics

\begin{tabular}{|c|l|}
\hline $\begin{array}{c}\text { Resultado do índice } \\
\mathbf{m}\end{array}$ & \multicolumn{1}{c|}{ Definição } \\
\hline $0<\mathrm{m} \leq 0,5$ & $\begin{array}{l}\text { Pode ser um tema de interesse para pesquisadores em um campo } \\
\text { específico de pesquisa, que engloba uma comunidade menor. }\end{array}$ \\
\hline $0,5<\mathrm{m} \leq 2$ & $\begin{array}{l}\text { Provavelmente pode se tornar um 'hot topic' como área de pesquisa, no } \\
\text { qual a comunidade é muito grande ou o tópico/combinação apresenta } \\
\text { características muito interessantes ou marcantes. }\end{array}$ \\
\hline $\mathrm{m}>2$ & $\begin{array}{l}\text { É considerado um 'hot topic', tópico exclusivo com alcance não apenas } \\
\text { na sua própria área de pesquisa. É provável que tenha efeitos de } \\
\text { aplicação ou características exclusivas. }\end{array}$ \\
\hline
\end{tabular}

Fonte: Elaborado com base em Banks (2006).

Portanto, os resultados serão analisados com base nas definições propostas por Banks (2006). Em síntese, as etapas para a análise dos resultados estão expostas no Quadro 2. 


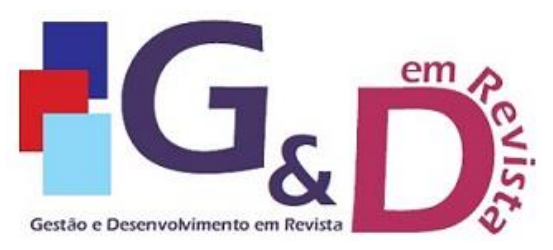

Gestão e Desenvolvimento em Revista V. 7, N. 1, jan-jun/2021, p. 71-81. ISSN online: $2446-8738$ Artigo recebido em: 27/05/2021 Artigo aprovado em: 16/08/2021

Quadro 2 - Síntese das etapas da análise dos resultados

\begin{tabular}{|l|l|}
\hline \multicolumn{1}{|c|}{ Etapas } & \multicolumn{1}{c|}{ Descrição } \\
\hline \multicolumn{1}{|c|}{} & - Áreas temáticas \\
& - Tipos de documentos \\
Características das publicações & - Ano das publicações \\
& - Autores \\
& - Títulos das fontes \\
& - Instituições \\
& - Agências financiadoras \\
& - Países \\
\hline Número de citações em cada publicação (análise dos hot topics) & - Idiomas \\
\hline
\end{tabular}

Fonte: Elaborado pelas autoras (2020).

Analisou-se as palavras-chave na indexação do trabalho da amostra, bem como os principais termos que puderam ser identificados nas publicações, retratando quais conceitos são trabalhados em conjunto e como eles se relacionam. Para esse fim, foi utilizado o software VOSviewer na formação de clusters de cocitação e de palavras-chave. Por fim, foram realizadas as análises dos hot-topics. A próxima seção apresenta a análise dos resultados do estudo.

\section{RESULTADOS E DISCUSSÕES}

\subsection{CARACTERÍSTICAS DAS PUBLICAÇÕES}

Com base nas 5.842 publicações encontradas, procedeu-se a análise das características das publicações. Inicialmente, apresenta-se os resultados acerca das áreas temáticas a partir das categorias da Web of Science (Quadro 3).

Quadro 3-Principais Áreas Temáticas

\begin{tabular}{|l|l|c|}
\hline \multicolumn{1}{|c|}{ Área Temática } & $\begin{array}{c}\text { Número de } \\
\text { publicações }\end{array}$ \\
\hline $1^{\circ}$ & Education Educational Research & 1440 \\
\hline $2^{\circ}$ & Environmental Sciences & 765 \\
\hline $3^{\circ}$ & Green Sustainable Science Technology & 650 \\
\hline $4^{\circ}$ & Environmental Studies & 365 \\
\hline $5^{\circ}$ & Management & 330 \\
\hline $6^{\circ}$ & Engineering Environmental & 327 \\
\hline $7^{\circ}$ & Education Scientific Disciplines & 298 \\
\hline $8^{\circ}$ & Public Environmental Occupational Health & 215 \\
\hline $9^{\circ}$ & Business & 210 \\
\hline $10^{\circ}$ & Engineering Multidisciplinary & 209 \\
\hline $11^{\circ}$ & Social Sciences Interdisciplinary & 203 \\
\hline $12^{\circ}$ & Engineering Electrical Electronic & 151 \\
\hline
\end{tabular}




\begin{tabular}{|l|l|c|}
\hline & \multicolumn{1}{|c|}{ Área Temática } & $\begin{array}{c}\text { Número de } \\
\text { publicações }\end{array}$ \\
\hline $13^{\circ}$ & Economics & 146 \\
\hline $14^{\circ}$ & Energy Fuels & 133 \\
\hline $15^{\circ}$ & Engineering Civil & 126 \\
\hline $16^{\circ}$ & Ecology & 121 \\
\hline $17^{\circ}$ & Water Resources & 119 \\
\hline $18^{\circ}$ & Information Science Library Science & 118 \\
\hline $19^{\circ}$ & Nursing & 106 \\
\hline $20^{\circ}$ & Computer Science Interdisciplinary Applications & 100 \\
\hline
\end{tabular}

Fonte: Elaborado pelas autoras (2020).

A maior concentração das publicações encontra-se na área temática Education Educational Research (Pesquisa Educacional), com 1440 publicações; a segunda maior área é a da temática Environmental Sciences (Ciências Ambientais), com 765 publicações; e, em terceiro, Green Sustainable Science Technology (Tecnologia e Ciências Sustentáveis Verde), que apresenta 650 publicações. Ainda, Environmental Studies (Estudos Ambientais), Management (Gestão), Education Scientific Disciplines (Disciplinas de Educação Científica) e Engineering Environmental (Engenharia Ambiental) são outras áreas com um número expressivo de publicações, que possuem foco em estudos relacionados ao meio ambiente. Pode-se inferir que a Pesquisa Educacional se destaca, pois optou-se por investigar as publicações relacionadas às Universidades e às IES. Ademais, Tecnologia e Ciências Sustentáveis Verde relaciona-se com o papel das IES acerca do desenvolvimento de tecnologia e de inovação. A seguir, o Quadro 4 apresenta os tipos de publicações.

Quadro 4 - Tipos de documentos

\begin{tabular}{|c|c|c|c|}
\hline & Tipos de documentos & $\begin{array}{l}\text { Número de } \\
\text { publicações }\end{array}$ & Frequência \\
\hline $1^{0}$ & Article & 3896 & $66,689 \%$ \\
\hline $2^{\circ}$ & Proceedings Paper & 1730 & $29,613 \%$ \\
\hline $3^{\circ}$ & Review & 201 & $3,441 \%$ \\
\hline $4^{\circ}$ & Editorial Material & 55 & $0,941 \%$ \\
\hline $5^{\circ}$ & Early Access & 10 & $0,171 \%$ \\
\hline $6^{\circ}$ & Biographical Item & 3 & $0,051 \%$ \\
\hline $7^{0}$ & Data Paper & 3 & $0,051 \%$ \\
\hline $8^{\circ}$ & Book Chapter & 2 & $0,034 \%$ \\
\hline $9^{\circ}$ & Book Review & 2 & $0,034 \%$ \\
\hline $10^{\circ}$ & Retracted Publication & 2 & $0,034 \%$ \\
\hline $11^{\circ}$ & Chronology & 1 & $0,017 \%$ \\
\hline $12^{0}$ & Correction & 1 & $0,017 \%$ \\
\hline $13^{0}$ & Letter & 1 & $0,017 \%$ \\
\hline $14^{\circ}$ & News Item & 1 & $0,017 \%$ \\
\hline
\end{tabular}

Fonte: Elaborado pelas autoras (2020). 


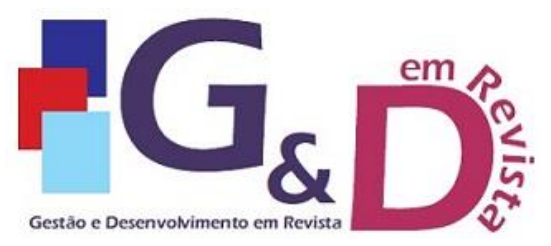

Gestão e Desenvolvimento em Revista V. 7, N. 1, jan-jun/2021, p. 71-81.

ISSN online: $2446-8738$

Artigo recebido em: 27/05/2021

Artigo aprovado em: 16/08/2021

Quanto aos tipos de documentos apresentados no Quadro 4, os artigos aparecem em destaque (66,689\%); em seguida, Proceedings Paper, com representatividade de $29,613 \%$, e Review, com 3,441\%. Na Figura 1, apresenta-se a quantidade de documentos por ano de publicação.

Figura 1 - Documentos por ano de publicação

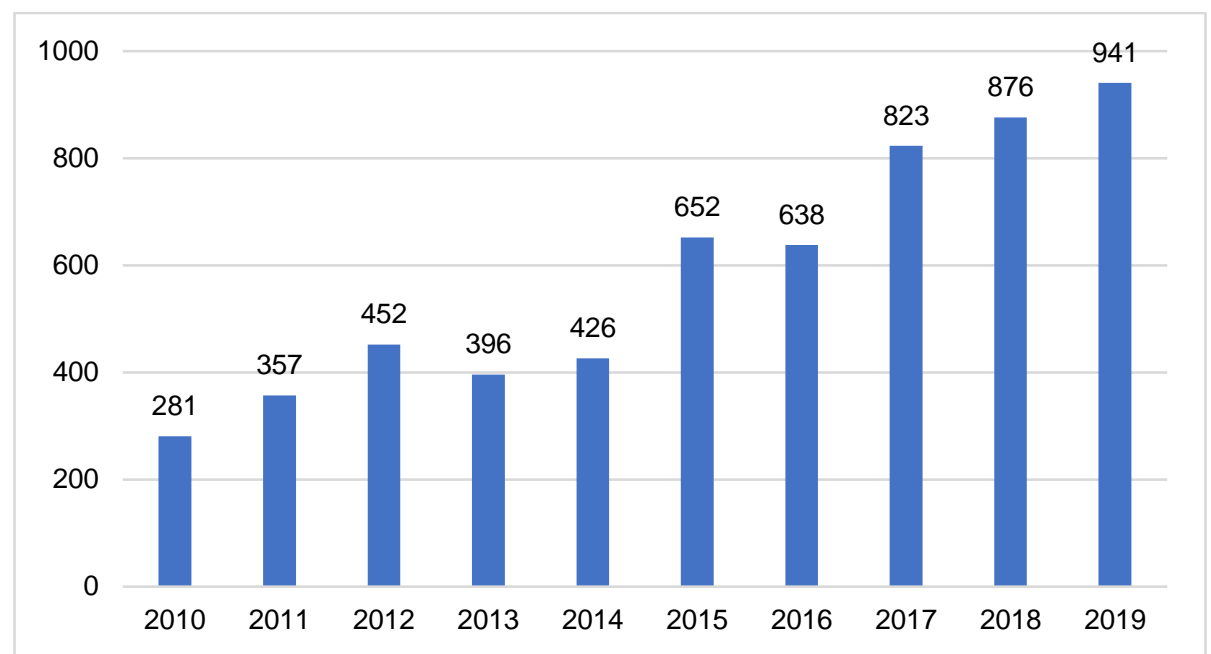

Fonte: Elaborado pelas autoras (2020).

Entre o período de 2017 a 2019, observa-se um aumento nas pesquisas sobre práticas sustentáveis e/ou gestão ambiental em universidades e IES, atingindo o auge em 2019. Os resultados indicam que o interesse nas publicações na temática vem crescendo, visto que as universidades, conforme apontam Amaral, Martins e Gouveia (2015), desempenham um papel importante quanto ao DS e devem servir de exemplo para outras organizações na inserção de práticas de sustentabilidade, bem como na disseminação do conhecimento. Os principais autores estão demonstrados no Quadro 5.

Fonte: Elaborado pelas autoras (2020).

Quadro 5- Principais autores e número de publicações
\begin{tabular}{|l|l|c|}
\hline & \multicolumn{1}{|c|}{ Autores } & Publicações \\
\hline $1^{\circ}$ & Donald Huisingh & 12 \\
\hline $2^{0}$ & Rodrigo Lozano & 10 \\
\hline $3^{0}$ & Walter Leal Filho & 9 \\
\hline $4^{\circ}$ & Yan Liu & 9 \\
\hline $5^{0}$ & Maria Paz Arraiza & 8 \\
\hline $6^{0}$ & Florin loras & 8 \\
\hline $7^{0}$ & Lei Liu & 8 \\
\hline
\end{tabular}


O autor que se destaca quanto às publicações sobre o tema é Donald Huisingh (University of Tennessee System), com 12 publicações, seguido de Rodrigo Lozano (University of Gavle), com 10 publicações, Walter Leal Filho (Hamburg University of Applied Sciences), com 9 publicações, e Yan Liu (Jining Medical University), com 9 publicações. Observa-se que não há grande diferença no número de publicações dos principais autores. Quanto às instituições, apresenta-se as principais no Quadro 6.

Quadro 6-Principais instituições

\begin{tabular}{|l|l|c|}
\hline \multicolumn{1}{|c|}{ Instituições } & Publicações \\
\hline $1^{\circ}$ & University of California System & 79 \\
\hline $2^{\circ}$ & University of North Carolina & 63 \\
\hline $3^{0}$ & State University System of Florida & 62 \\
\hline $4^{\circ}$ & Universidade de São Paulo & 54 \\
\hline $5^{\circ}$ & Pennsylvania Commonwealth System of Higher Education Pcshe & 48 \\
\hline $6^{\circ}$ & University of London & 46 \\
\hline $7^{0}$ & University of Texas System & 43 \\
\hline $8^{\circ}$ & United States Department of Agriculture USDA & 41 \\
\hline $9^{0}$ & University of Wisconsin System & 40 \\
\hline $10^{\circ}$ & University of Minnesota System & 39 \\
\hline $11^{\circ}$ & Universitat Politecnica de Valencia & 38 \\
\hline $12^{\circ}$ & University of British Columbia & 38 \\
\hline $13^{\circ}$ & Michigan State University & 37 \\
\hline $14^{\circ}$ & University of Florida & 37 \\
\hline $15^{\circ}$ & University of Melbourne & 35 \\
\hline $16^{\circ}$ & University of Minnesota Twin Cities & 34 \\
\hline $17^{\circ}$ & University System of Georgia & 33 \\
\hline $18^{\circ}$ & University of Illinois System & 32 \\
\hline $19^{\circ}$ & Griffith University & 32 \\
\hline $20^{\circ}$ & Universidad Politecnica de Madrid & \\
\hline
\end{tabular}

Fonte: Elaborado pelas autoras (2020).

As três instituições com maior destaque nas publicações são a University of California System, com 79 publicações, a University of North Carolina, com 63 publicações, e a State University System of Florida, com 62 publicações, todas localizadas nos Estados Unidos da América. A Universidade de São Paulo (Brasil) aparece como quarta instituição, com 54 publicações. As principais fontes de publicação são apresentadas no Quadro 7.

Quadro 7- Principais fontes de publicação

\begin{tabular}{|l|l|c|}
\hline & \multicolumn{1}{|c|}{ Fontes } & Publicações \\
\hline $1^{\circ}$ & Journal of Cleaner Production & 191 \\
\hline $2^{\circ}$ & Edulearn Proceedings & 144 \\
\hline $3^{\circ}$ & International Journal of Sustainability in Higher Education & 141 \\
\hline $4^{\circ}$ & Sustainability & 137 \\
\hline $5^{\circ}$ & Inted Proceedings & 130 \\
\hline
\end{tabular}




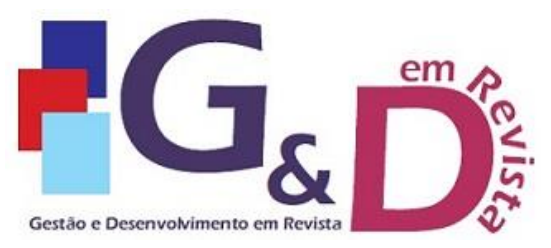

Gestão e Desenvolvimento em Revista V. 7, N. 1, jan-jun/2021, p. 71-81.

ISSN online: $2446-8738$

Artigo recebido em: 27/05/2021

Artigo aprovado em: 16/08/2021

\begin{tabular}{|c|c|c|}
\hline & Fontes & Publicações \\
\hline $6^{\circ}$ & Iceri Proceedings & 94 \\
\hline $7^{\circ}$ & Procedia Social and Behavioral Sciences & 80 \\
\hline $8^{\circ}$ & Asee Annual Conference Exposition & 60 \\
\hline 9o & Advances in Social Science Education And Humanities Research & 37 \\
\hline $10^{\circ}$ & Acta Horticulturae & 33 \\
\hline 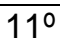 & Procedia Environmental Sciences & 33 \\
\hline $12^{0}$ & Bmj Open & 29 \\
\hline $13^{\circ}$ & $\begin{array}{l}\text { Edulearn } 19 \text { 11th International Conference on Education and New Learning } \\
\text { Technologies }\end{array}$ & 29 \\
\hline $14^{\circ}$ & $\begin{array}{l}\text { Towards Green Campus Operations Energy Climate and Sustainable } \\
\text { Development Initiatives at Universities }\end{array}$ & 28 \\
\hline $15^{0}$ & World Sustainability Series & 28 \\
\hline $16^{\circ}$ & Procedia Engineering & 24 \\
\hline $17^{\circ}$ & 10th International Conference of Education Research and Innovation Iceri 2017 & 22 \\
\hline $18^{0}$ & 12th International Technology Education and Development Conference Inted & 22 \\
\hline $19^{\circ}$ & $\begin{array}{l}\text { Edulearn } 18 \text { 10th International Conference on Education and New Learning } \\
\text { Technologies }\end{array}$ & 22 \\
\hline $20^{\circ}$ & $\begin{array}{lllllll}\text { Inted } 2014 \text { 8th International Technology Education and Development } \\
\text { Conference }\end{array}$ & 22 \\
\hline
\end{tabular}

Fonte: Elaborado pelas autoras (2020).

Os periódicos com maior destaque são o Journal of Cleaner Production, o Edulearn Proceedings e o International Journal of Sustainability in Higher Education e Sustainability, todos com foco em pesquisas sobre a temática da sustentabilidade. O International Journal of Sustainability in Higher Education publica estudos e tendências em sustentabilidade em um contexto de ensino superior. Nesse sentido, destaca-se que as IES assumem papel de protagonistas quanto ao Desenvolvimento Sustentável, bem como na implementação e na conscientização dos ODS.

Destaca-se, também, com um número expressivo de publicações (130), a Inted Proceedings (Conferência Internacional anual de Tecnologia, Educação e Desenvolvimento). Acerca das agências financiadoras, os resultados são listados no Quadro 8.

Quadro 8-Agências de financiamento

\begin{tabular}{|l|l|c|}
\hline \multicolumn{1}{|c|}{ Agências de financiamento } & Registros \\
\hline $1^{\circ}$ & United States Department of Health Human Services & 93 \\
\hline $2^{\circ}$ & National Institutes of Health Nih USA & 81 \\
\hline $3^{\circ}$ & National Natural Science Foundation of China NSFC & 56 \\
\hline $4^{\circ}$ & National Science Foundation NSF & 56 \\
\hline $5^{0}$ & European Union Eu & 42 \\
\hline $6^{0}$ & Ministry of Education Culture Sports Science and Technology Japan Mext & 27 \\
\hline $7^{0}$ & National Council for Scientific and Technological Development CNPQ & 27 \\
\hline $8^{\circ}$ & Capes & 24 \\
\hline $9^{\circ}$ & United States Department Of Agriculture Usda & 23 \\
\hline $10^{\circ}$ & Australian Research Council & 18 \\
\hline $11^{\circ}$ & European Commission Joint Research Centre & 16 \\
\hline $12^{\circ}$ & Japan Society for The Promotion of Science & 16 \\
\hline
\end{tabular}




\begin{tabular}{|c|l|c|}
\hline & \multicolumn{1}{|c|}{ Agências de financiamento } & Registros \\
\hline $13^{\circ}$ & Portuguese Foundation For Science and Technology & 16 \\
\hline $14^{\circ}$ & Natural Sciences and Engineering Research Council of Canada & 14 \\
\hline $15^{\circ}$ & United States Environmental Protection Agency & 14 \\
\hline $16^{\circ}$ & Social Sciences and Humanities Research Council of Canada Sshrc & 12 \\
\hline $17^{\circ}$ & Australian Government & 11 \\
\hline $18^{\circ}$ & Economic Social Research Council ESRC & 11 \\
\hline $19^{\circ}$ & Engineering Physical Sciences Research Council EPSRC & 11 \\
\hline $20^{\circ}$ & Ministry of Education Universities and Research Miur & 11 \\
\hline
\end{tabular}

Fonte: Elaborado pelas autoras (2020).

Em relação às agências de fomento, a United States Department of Health Human Services apresentou 93 registros, seguida da National Institutes of Health Nih USA, com 81 registros, ambas dos Estados Unidos da América; logo após, aparecem a National Natural Science Foundation of China (NSFC) da China e a National Science Foundation (NSF), ambas com 56 registros. Capes e CNPq não aparecem bem colocadas, salientando que os investimentos são limitados. No Quadro 9, apresenta-se os resultados acerca dos países que possuem publicações em relação ao tema pesquisado.

Quadro 9- Principais países

\begin{tabular}{|l|l|r|r|l|r|}
\hline & Países & $\begin{array}{l}\text { Número de } \\
\text { Publicações }\end{array}$ & & Países & $\begin{array}{l}\text { Número de } \\
\text { Publicações }\end{array}$ \\
\hline $1^{\circ}$ & Estados Unidos da América & 1.338 & $11^{\circ}$ & África do Sul & 147 \\
\hline $2^{\circ}$ & Inglaterra & 437 & $12^{\circ}$ & Romênia & 128 \\
\hline $3^{\circ}$ & China & 408 & $13^{\circ}$ & Holanda & 113 \\
\hline $4^{\circ}$ & Austrália & 392 & $14^{\circ}$ & Índia & 111 \\
\hline $5^{\circ}$ & Espanha & 392 & $15^{\circ}$ & Rússia & 107 \\
\hline $6^{\circ}$ & Brasil & 318 & $16^{\circ}$ & Suécia & 102 \\
\hline $7^{\circ}$ & Canadá & 257 & $17^{\circ}$ & Japão & 101 \\
\hline $8^{\circ}$ & Itália & 257 & $18^{\circ}$ & Portugal & 98 \\
\hline $9^{\circ}$ & Alemanha & 199 & $19^{\circ}$ & França & 94 \\
\hline $10^{\circ}$ & Malásia & 175 & $20^{\circ}$ & Turquia & 94 \\
\hline
\end{tabular}

Fonte: Elaborado pelas autoras (2020).

O Quadro 9 apresenta como principais países em número de publicações os Estados Unidos da América, a Inglaterra e a China. Em seguida, aparecem a Austrália, a Espanha e o Brasil (6º). Observa-se que o Brasil aparece como um dos principais países, com 318 publicações, fator positivo a fim de incentivar a ampliação de publicações na temática. Quanto aos idiomas, aparece em destaque o inglês, com 5.420 registros, seguido do espanhol, com 180 publicações, e do português, com 113 publicações. Na sequência, apresenta-se o relatório de citações por ano a partir das publicações (Figura 2). 
Figura 2 - Relatório de citações

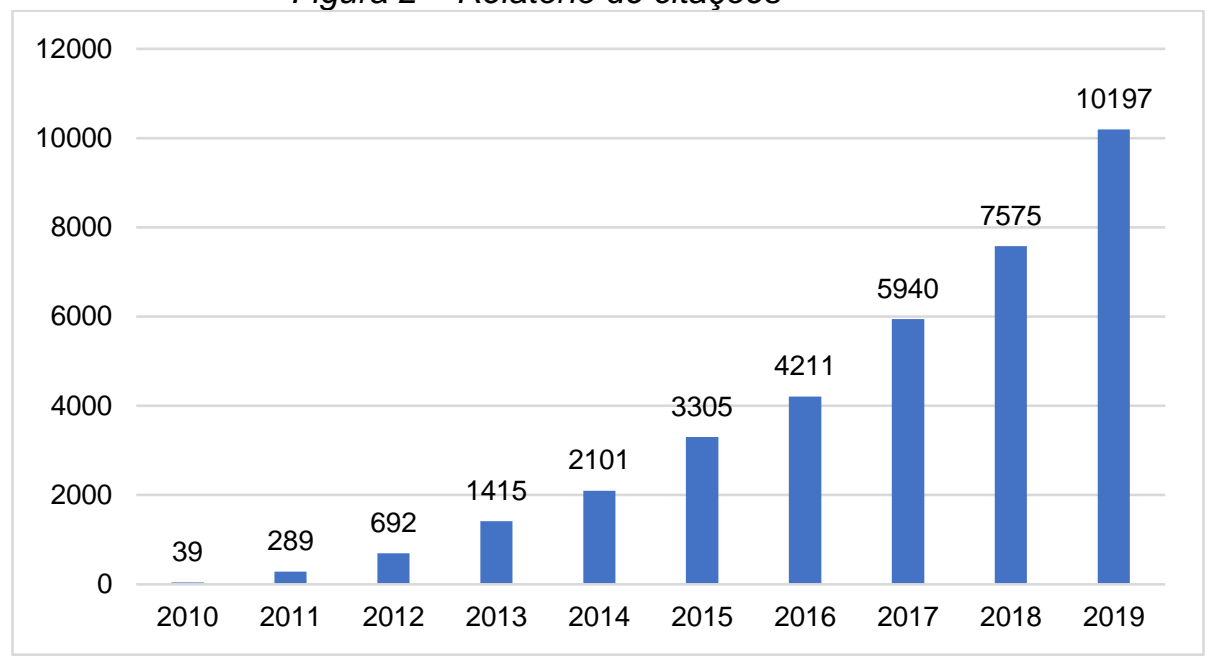

Fonte: Web of Science (2020).

O período apurado totalizou 55.490 citações e, destas, 53.036 são citações sem autocitações. A média de citações por item é de 9,39 e o h-index é de 82. Um índice de $h$ significa que há publicações $H$ que foram citados pelo menos $h$ vezes.

O relatório de citações informa o h (o número de artigos publicados e a frequência com que esses artigos são citados). Por meio da análise de citações, identificou-se os trabalhos mais citados sobre a temática (Quadro 10).

\begin{tabular}{|c|c|c|}
\hline Descrição & $\begin{array}{c}\text { Ano da } \\
\text { Publicação }\end{array}$ & Citações \\
\hline $\begin{array}{l}\text { Culturally Sustaining Pedagogy: A Needed Change in Stance, } \\
\text { Terminology, and Practice. Por: Paris, Django. EDUCATIONAL } \\
\text { RESEARCHER Volume: } 41 \\
\text { Edição: } 3 \text { Páginas: } 93-97 .\end{array}$ & 2012 & 776 \\
\hline $\begin{array}{l}\text { Soil salinity: A serious environmental issue and plant growth- } \\
\text { promoting bacteria as one of the tools for its alleviation } \\
\text { Por: Shrivastava, Pooja; Kumar, Rajesh. SAUDI JOURNAL OF } \\
\text { BIOLOGICAL SCIENCES Volume: } 22 \text { Edição: } 2 \text { Páginas: 123- } \\
\begin{array}{llll}131 . & \end{array}\end{array}$ & 2015 & 641 \\
\hline $\begin{array}{l}\text { Environmental innovation and R\&D cooperation: } \\
\text { evidence from Spanish manufacturing firms. Por: De Marchi, } \\
\text { Valentina. RESEARCH POLICY Volume: } 41 \text { Edição: } 3 \text { Páginas: } \\
\text { 614-623. }\end{array}$ & 2012 & 465 \\
\hline $\begin{array}{l}\text { Transdisciplinarity: Between mainstreaming and marginalization. } \\
\text { Por: Jahn, Thomas; Bergmann, Matthias; Keil, Florian. ECOLOGICAL } \\
\text { ECONOMICS Volume: } 79 \text { Páginas: } 1-10 \text {. }\end{array}$ & 2012 & 395 \\
\hline $\begin{array}{l}\text { A review of commitment and implementation of sustainable } \\
\text { development in higher education: results from a worldwide survey. } \\
\text { Por: Lozano, Rodrigo; Ceulemans, Kim; Alonso-Almeida, Mar; et al. } \\
\text { JOURNAL OF CLEANER PRODUCTION Volume: } 108 \text { Páginas: 1- } \\
18 .\end{array}$ & 2015 & 276 \\
\hline
\end{tabular}




\begin{tabular}{|c|c|c|}
\hline Descrição & $\begin{array}{c}\text { Ano da } \\
\text { Publicação }\end{array}$ & Citações \\
\hline $\begin{array}{l}\text { Drivers of different types of eco-innovation in European SMEs. } \\
\text { Por: Triguero, Angela; Moreno-Mondejar, Lourdes; Davia, Maria A. } \\
\text { ECOLOGICAL ECONOMICS Volume: } 92 \text { Edição especial: } \\
\text { SI Páginas: } 25-33 \text {. }\end{array}$ & 2013 & 275 \\
\hline 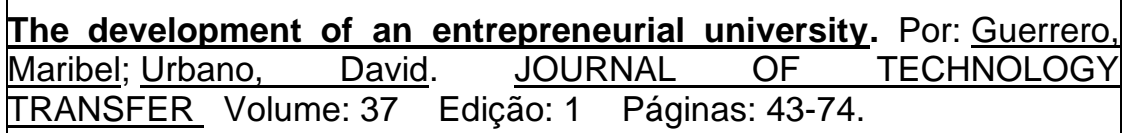 & 2012 & 253 \\
\hline $\begin{array}{l}\text { Achieving Research Impact Through Co-creation in Community- } \\
\text { Based Health Services: Literature Review and Case Study. } \\
\text { Por: Greenhalgh, Trisha; Jackson, Claire; Shaw, Sara; et al. } \\
\text { MILBANK QUARTERLY } \\
\text { Volume: } 94 \quad \text { Edicão: } 2 \text { Páqinas: } 392-429 .\end{array}$ & 2016 & 230 \\
\hline 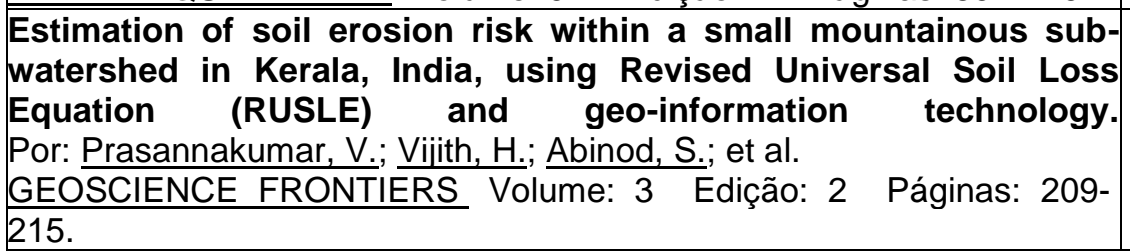 & 2012 & 219 \\
\hline 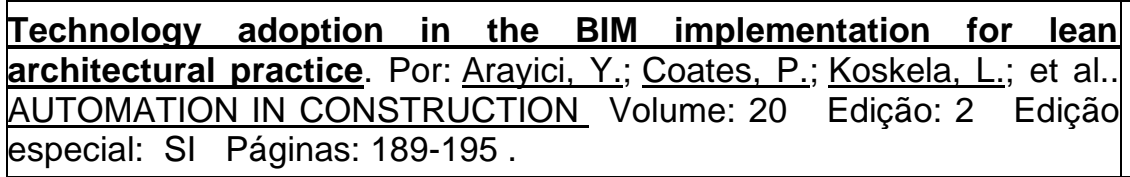 & 2011 & 215 \\
\hline
\end{tabular}

Fonte: Web of Science (2020).

Dentre as publicações mais citadas, tem-se o estudo "Culturally Sustaining Pedagogy: A Needed Change in Stance, Terminology, and Practice", do autor Paris (2012), com um total de 776 citações no período desde a data de publicação, no ano de 2012, até o presente momento. A partir de um ensaio teórico, o autor oferece o termo e a postura da pedagogia culturalmente sustentável como uma alternativa elencada para a melhora das pesquisas voltadas à temática e às práticas.

A seguir, apresenta-se o mapa de cocitações dos autores (Figura 3), o qual permite analisar os clusters existentes e os autores que pertencem a eles, podendo, dessa forma, prospectar pares de autores citados em conjunto, em um mesmo estudo ou com teorias em comum (RAMOS-RODRÍGUEZ; RUÍZ-NAVARRO, 2004). Foram selecionados autores citados pelo menos 20 vezes, verificando os possíveis grupos ou pares de artigos que são citados em conjunto com outro artigo. 
Figura 3-Relatório de cocitações

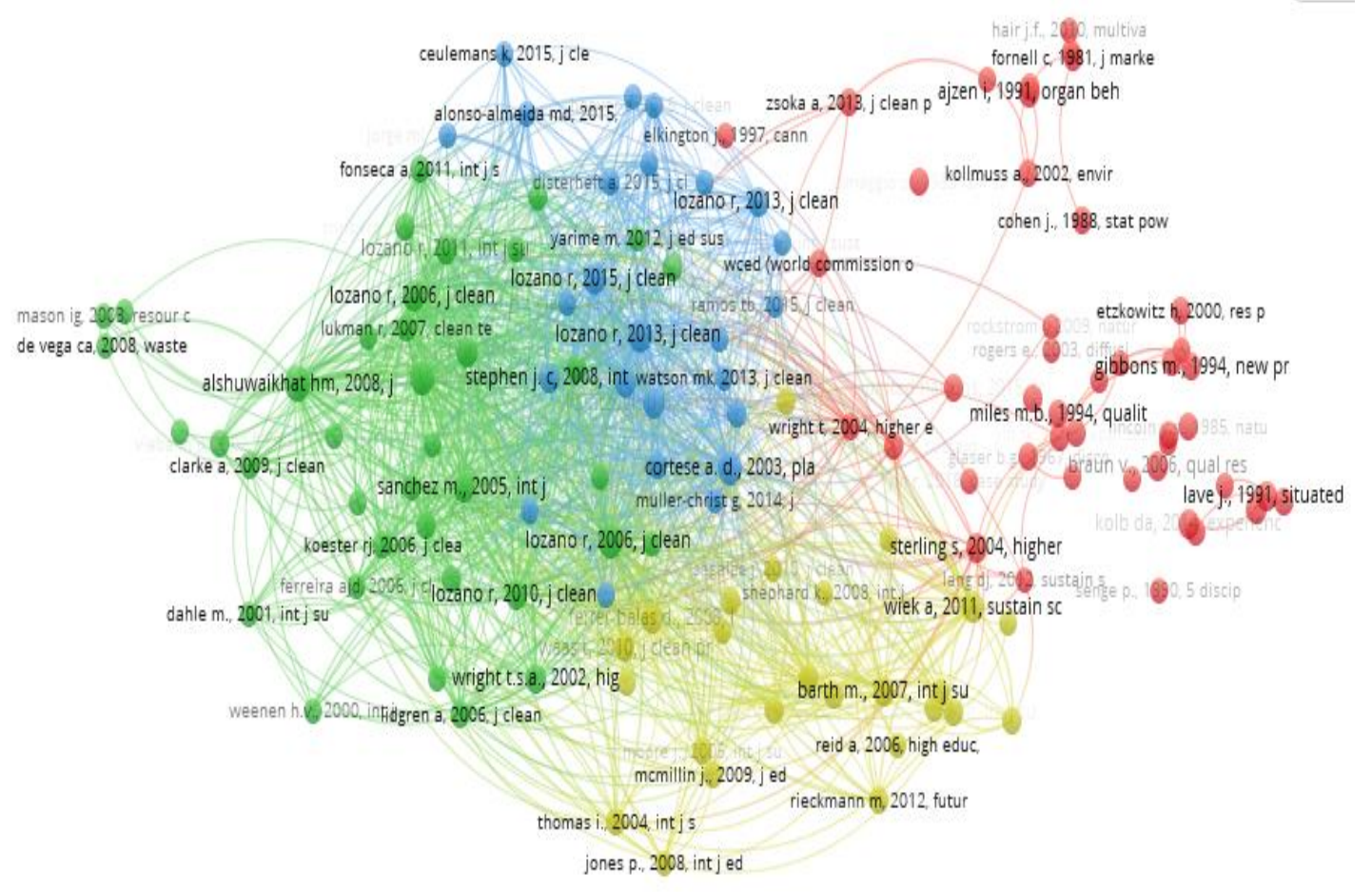

Fonte: Elaborado pelas autoras por meio do VOSViewer.

Dos 176.802 links formados pelas referências cocitadas, 132 atenderam ao limite de 20 vezes como número mínimo de cocitações. Em primeiro está Lozano (2013), com 110 citações. Em segundo lugar, o autor Velazquez (2006) aparece com 100 citações e, em terceiro lugar, o autor Cortese (2003), com 99 cocitações. Assim, na figura 4 tem-se 4 clusters com total de 132 autores, sendo que o maior cluster é o vermelho, com 44 autores, dentre eles Elkington (1997), Etzkowit (2000), Fornell (1981) e Kolb (2014). Já o segundo cluster, de cor verde, é composto por 38 autores, entre eles Lozano (2006), Lukman (2007) e Yuan (2013). O terceiro cluster, de cor azul, é composto por 25 autores; por fim, o quarto cluster, de cor amarela, também apresenta 25 autores. A Figura 4 demonstra o mapa textual das palavras chaves das 5.842 publicações encontradas. 


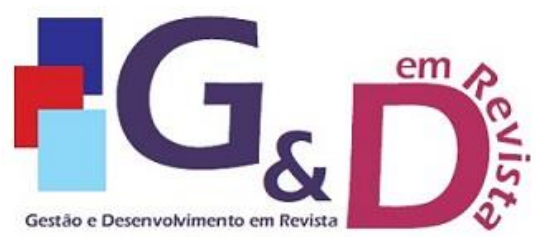

Gestão e Desenvolvimento em Revista V. 7, N. 1, jan-jun/2021, p. 71-81.

ISSN online: $2446-8738$

Artigo recebido em: 27/05/2021

Artigo aprovado em: 16/08/2021

Figura 4 - Mapa textual - rede de palavras da WOS

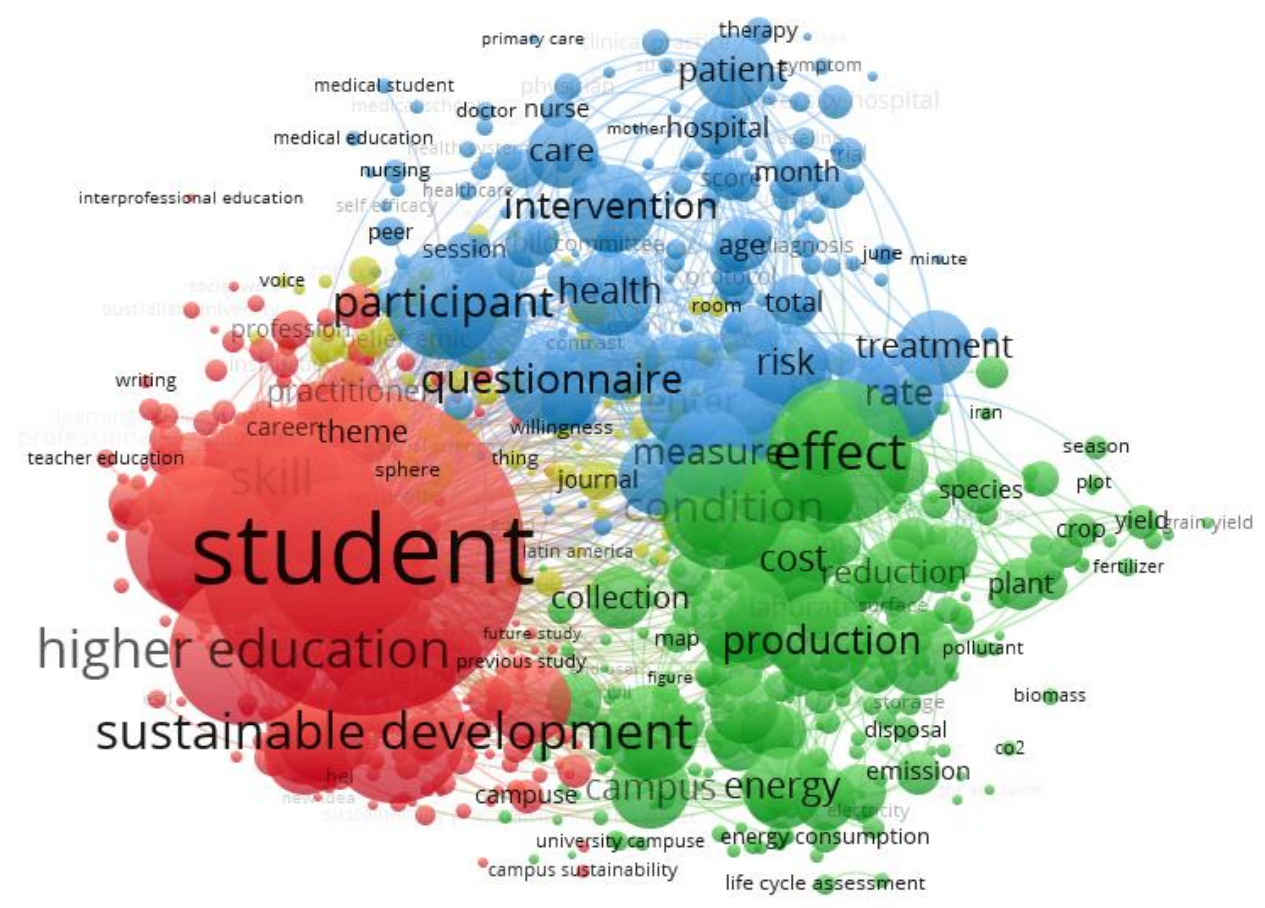

Fonte: Elaborado pelas autores por meio do Vosviewer.

As análises dos termos ou das palavras-chave mais citados foram realizadas utilizando o software VOSViewer. Inicialmente, foram selecionados os termos com mais de 20 ocorrências. A partir de então, o software identificou um total de 125.722 termos ou palavras-chave. Destes, 1.571 apresentam mais de 20 ocorrências.

Elaborou-se uma rede com as palavras-chave mais adotadas (Figura 4). Nessa rede, verificam-se quatro clusters predominantes, sendo que o principal corresponde às palavras elencadas no grupo 'student', em vermelho, com 331 itens, onde estão trabalhos voltados ao ensino superior e ao desenvolvimento sustentável. Observa-se o cluster de cor verde composto por 309 palavras-chave com o termo 'effect' como o principal expoente. No cluster de cor azul, constam 189 termos com estudos voltados para a área médica e suas intervenções e tratamentos, prevenções e protocolos, bem como estudos voltados a fatores de riscos e instituições e a programas voltados à saúde em um contexto de sustentabilidade. E, por fim, o cluster de cor amarelo é composto por 114 palavras-chave ligadas a pesquisas voltadas para estudos e políticas governamentais no setor das ciências sociais e da saúde pública. Na próxima seção, serão analisados os hot topics. 


\subsection{ANÁLISE DOS HOT TOPICS}

Após a análise das características das publicações, prosseguiu-se com o exame dos hot topics. A partir da busca na Web of Science da combinação dos termos práticas sustentáveis (sustainable practices) ou gestão ambiental (environmental management) e universidades (universities) ou instituições de ensino superior (higher education institutions), procedeu-se a busca para cada tópico relacionado à área temática, em que foram calculados os índices h-b e m (BANKS, 2006) conforme apresentado no Quadro 11.

Quadro 11- Hot Topics (tópicos quentes) e índices ' $h$-b' e ' $m$ '

\begin{tabular}{|c|c|c|c|c|}
\hline & Área Temática & $\begin{array}{l}\text { Número de } \\
\text { publicações }\end{array}$ & $\begin{array}{c}\text { Îndice } \\
\text { h-b }\end{array}$ & $\begin{array}{c}\text { Îndice } \\
\text { m }\end{array}$ \\
\hline $1^{0}$ & Management (Gestão) & 3244 & 62 & 6,2 \\
\hline $2^{0}$ & Environmental Studies (Estudos Ambientais) & 1720 & 48 & 4,8 \\
\hline $3^{\circ}$ & Environmental Sciences (Ciências Ambientais) & 564 & 30 & 3,0 \\
\hline $4^{\circ}$ & Business (Negócios) & 561 & 30 & 3,0 \\
\hline $5^{\circ}$ & $\begin{array}{l}\text { Education Educational Research (Pesquisa na } \\
\text { Educação) }\end{array}$ & 428 & 27 & 2,7 \\
\hline 60 & $\begin{array}{lll}\begin{array}{l}\text { Engineering } \\
\text { Ambiental) }\end{array} & \text { Environmental } & \text { (Engenharia } \\
\end{array}$ & 442 & 26 & 2,6 \\
\hline $7^{0}$ & Nursing (Enfermagem) & 193 & 21 & 2,1 \\
\hline $8^{\circ}$ & Water Resources & 221 & 20 & 2,0 \\
\hline 9운 & Ecology & 136 & 17 & 1,7 \\
\hline $10^{\circ}$ & Economics & 136 & 16 & 1,6 \\
\hline $11^{\circ}$ & Social Sciences Interdisciplinary & 49 & 13 & 1,3 \\
\hline $12^{0}$ & Energy Fuels & 70 & 12 & 1,2 \\
\hline $13^{0}$ & Engineering Civil & 112 & 11 & 1,1 \\
\hline $14^{\circ}$ & Health Care Sciences Services & 23 & 9 & 0,9 \\
\hline $15^{\circ}$ & Engineering Multidisciplinary & 43 & 7 & 0,7 \\
\hline $16^{\circ}$ & Multidisciplinary Sciences & 45 & 7 & 0,7 \\
\hline $17^{\circ}$ & Regional Urban Planning & 21 & 6 & 0,6 \\
\hline $18^{\circ}$ & Education Scientific Disciplines & 32 & 5 & 0,5 \\
\hline $19^{\circ}$ & Green Sustainable Science Technology & 20 & 4 & 0,4 \\
\hline $20^{\circ}$ & Public Environmental Occupational Health & 9 & 4 & 0,4 \\
\hline
\end{tabular}

Fonte: Elaborado pelas autoras (2020).

$\mathrm{Na}$ análise dos hot topics, foi possível constatar que as áreas temáticas Management (Gestão), Environmental Studies (Estudos Ambientais), Environmental Sciences (Ciências Ambientais), Business (Negócios), Education Educational Research (Pesquisa na Educação), Engineering Environmental (Engenharia Ambiental) e Nursing (Enfermagem) apresentaram índice $\mathrm{m}>2$, sendo, portanto, consideradas hot topics (BANKS, 2006). 
Nesse ínterim, pode-se inferir que as duas correntes de desenvolvimento sustentável nas universidades estão presentes na produção científica: (1) a questão educacional (acadêmica) e (2) as iniciativas de implementação da gestão ambiental nas IES (TAUCHEN; BRANDLI, 2006). Outrossim, a área de Gestão oferece insights para pesquisas relacionadas à proposição e à aplicação de modelos, à gestão de resíduos em IES e às práticas de gestão ambiental (eixo operacional), enquanto as áreas 'Estudos Ambientais', 'Ciências Ambientais' e 'Pesquisa na Educação', evidenciam a importância de estudos relacionados ao eixo acadêmico (ROHRICH; TAKAHASHI, 2019).

Por conseguinte, os resultados indicam a importância das áreas temáticas na pesquisa sobre sustentabilidade e gestão ambiental nas IES, de modo que demonstrar políticas e práticas de governança e gestão, a inserção do Desenvolvimento Sustentável na formação acadêmica e propor indicadores e instrumentos de avaliação da Gestão Ambiental nas universidades contribui com o conhecimento científico e para a divulgação de modelos que poderão ser replicados em outras organizações.

\section{CONSIDERAÇÕES FINAIS}

Este estudo teve por objetivo apresentar um panorama da produção científica sobre práticas sustentáveis e gestão ambiental em Instituições de Ensino Superior na base de dados Web of Science, no período de 2010 a 2019, bem como identificar os hot topics relacionados à temática estudada. A partir do estudo bibliométrico, foi possível identificar 5.842 publicações para o período em estudo, sendo 3.896 artigos (66,68\%).

As áreas temáticas com maior concentração de publicações são Education Educational Research (Pesquisa Educacional), Environmental Sciences (Ciências Ambientais) e Green Sustainable Science Technology (Tecnologia e Ciências Sustentáveis Verde). Enquanto em 2010 foram identificadas apenas 210 publicações, no período de 2017-2019 observa-se um aumento nas pesquisas, sendo que atingiram o auge em 2019. No maior cluster de autores figura Elkington (1997), Etzkowit (2000), Fornell (1981) e Kolb (2014). 
As três instituições com maior destaque nas publicações são a University of California System, a University of North Carolina e a State University System of Florida, todas localizadas nos Estados Unidos. Enfatiza-se a Universidade de São Paulo em 6 lugar, com 54 publicações na área. Os periódicos com maior destaque são o Journal of Cleaner Production, o Edulearn Proceedings e o International Journal of Sustainability in Higher Education e Sustainability, todos com foco em pesquisas sobre a temática da sustentabilidade.

A partir das análises dos índices 'h-b' e 'm', constatou-se que as áreas de Management (Gestão), Environmental Studies (Estudos Ambientais), Environmental Sciences (Ciências Ambientais), Business (Negócios), Education Educational Research (Pesquisa na Educação), Engineering Environmental (Engenharia Ambiental) e Nursing (Enfermagem) são hot topics (tópicos quentes) para a pesquisa sobre práticas sustentáveis e gestão ambiental em Instituições de Ensino Superior.

Importante ressaltar o papel de protagonismo das universidades na sociedade e a necessidade de divulgação das práticas ambientais tanto no meio acadêmico quanto entre as organizações, como incentivo para aprimorar as decisões estratégicas da gestão, melhorar o relacionamento da universidade com seus stakeholders (aumentando investimentos e/ou financiamentos em projetos), criar valor sustentável para a universidade e permitir o alcance de objetivos sociais, ambientais e econômicos. Os eixos acadêmico e de gestão devem atuar de forma integrada para o fortalecimento da Gestão Ambiental, permitindo o alcance das metas da organização de forma eficiente e eficaz.

Como limitação, meciona-se a utilização de apenas uma base de dados. Para estudos futuros, sugere-se a análise qualitativa das publicações sobre o tema, incluindo outras bases de dados.

\section{REFERÊNCIAS}

AMARAL, Luís P.; MARTINS, Nelson; GOUVEIA, Joaquim B. Quest for a sustainable university: a review. International Journal of Sustainability in Higher Education, 2015.

ARIVUMANI, A.; VIJAYALAXMI I. Sustainable Development. Advances in Natural and Applied Sciences. 11(7). 296-300. 2017. 


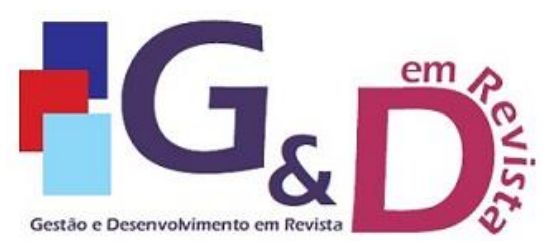

Gestão e Desenvolvimento em Revista V. 7, N. 1, jan-jun/2021, p. 71-81.

ISSN online: $2446-8738$

Artigo recebido em: 27/05/2021

Artigo aprovado em: 16/08/2021

ÁVILA, Lucas Veiga et al. Barriers to innovation and sustainability at universities around the world. Journal of cleaner production, v. 164, p. 1268-1278, 2017.

BANKS, Michael G. An extension of the Hirsch index: Indexing scientific topics and compounds. Scientometrics, v. 69, n. 1, p. 161-168, 2006.

BRUNDTLAND, Gro Harlem et al. Nosso futuro comum. Rio de Janeiro: FGV, v. 2, 1991.

CAIADO, Rodrigo Goyannes Gusmão et al. A literature-based review on potentials and constraints in the implementation of the sustainable development goals. Journal of cleaner production, v. 198, p. 1276-1288, 2018.

\section{CAPES. COORDENAÇÃO DE APERFEIÇOAMENTO DE PESSOAL DA NÍVEL}

SUPERIOR. Disponível em: <

https://www.periodicos.capes.gov.br/?option=com_pcollection\&mn=70\&smn=79\&cid $=81>$. Acesso em: 20 setembro. 2020 .

DAGILIŪTĖ, Renata; LIOBIKIENĖ, Genovaitè; MINELGAITĖ, Audronè. Sustainability at universities: Students' perceptions from Green and Non-Green

universities. Journal of Cleaner Production, v. 181, p. 473-482, 2018.

DISTERHEFT, Antje et al. Environmental Management Systems (EMS)

implementation processes and practices in European higher education institutionsTop-down versus participatory approaches. Journal of Cleaner Production, v. 31, p. 80-90, 2012.

ELKINGTON, John. Canibais com garfo e faca. São Paulo: Makron Books, 2001.

GLASER, Gisbert. Base sustainable development goals on science. Nature, v. 491, n. 7422, p. 35-35, 2012.

HAIR, Joseph et al. Fundamentos de métodos de pesquisa em administração. Porto Alegre: Bookman, 2005.

HALL, Rosemar José et al. Compras públicas sustentáveis: um estudo nas universidades federais brasileiras. Amazônia, Organizações e Sustentabilidade, v. 3, n. 1, p. 27-44, 2014.

HOVE, Hilary. Critiquing sustainable development: a meaningful way of mediating the development impasse?. Undercurrent, v. 1, n. 1, 2004.

JORGE, Manuel Larrán et al. An approach to the implementation of sustainability practices in Spanish universities. Journal of Cleaner Production, v. 106, p. 34-44, 2015.

LARA, José Edson et al. Adoção dos critérios de sustentabilidade ambiental no processo de compras de uma Universidade Federal. Revista IPTEC, v. 3, n. 2, p. 254-267, 2015.

LEAL FILHO, Walter et al. Sustainable development policies as indicators and preconditions for sustainability efforts at universities: fact or fiction?. International Journal of Sustainability in Higher Education, 2018.

LEAL FILHO, Walter et al. The role of planning in implementing sustainable development in a higher education context. Journal of cleaner production, v. 235, p. 678-687, 2019. 


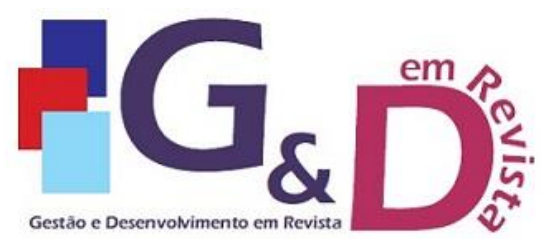

Gestão e Desenvolvimento em Revista V. 7, N. 1, jan-jun/2021, p. 71-81.

ISSN online: $2446-8738$

Artigo recebido em: 27/05/2021

Artigo aprovado em: 16/08/2021

LOZANO, Rodrigo et al. Declarations for sustainability in higher education: becoming better leaders, through addressing the university system. Journal of Cleaner Production, v. 48, p. 10-19, 2013.

MACEDO, Marcelo Alvaro da Silva; CASA NOVA, Silvia Pereira de Castro; ALMEIDA, Katia de. Mapeamento e análise bibliométrica da utilização da Análise Envoltória de Dados (DEA) em estudos em contabilidade e administração. Contabilidade, Gestão e Governança, v. 12, n. 3, 2010.

ONU. Organização das Nações Unidas. Os Objetivos de Desenvolvimento Sustentável no Brasil. Disponível em :<https://brasil.un.org/pt-br/sdgs>. Acesso em: 20 junho. 2021.

PARIS, Django. Culturally sustaining pedagogy: A needed change in stance, terminology, and practice. Educational researcher, v. 41, n. 3, p. 93-97, 2012.

RAGAZZI, Marco; GHIDINI, Francesca. Environmental sustainability of universities: critical analysis of a green ranking. Energy Procedia, v. 119, p. 111-120, 2017.

RAMOS-RODRÍGUEZ, Antonio-Rafael; RUÍZ-NAVARRO, José. Changes in the intellectual structure of strategic management research: A bibliometric study of the Strategic Management Journal, 1980-2000. Strategic management journal, v. 25, n. 10, p. 981-1004, 2004.

ROHRICH, Sandra Simm; TAKAHASHI, Adriana Roseli Wünsch. Sustentabilidade ambiental em Instituições de Ensino Superior, um estudo bibliométrico sobre as publicações nacionais. Gestão \& Produção, v. 26, 2019.

ROSTAING, Hervé. La bibliométrie et es techniques. Tolouse: Sciences de la Société, 1997.

RUIZ-MALLÉN, I.; HERAS, M. What sustainability? Higher education institutions' pathways to reach the Agenda 2030 goals. Sustainability, v. 12, n. 4, p. 1290, 2020.

SAYED, Abu; KAMAL, Md.; ASMUSS, Margret. Benchmarking tools for assessing and tracking sustainability in higher educational institutions. International Journal of Sustainability in Higher Education, 2013, 14(4), 449-465.

SILVA, Márcia Regina da. Análise biliométrica da produção científica docente do Programa de Pós-Graduação em Educação Especial da UFSCar: 1998-2003. 2004.

SILVA, Gilberto Soares da; AZEVEDO, Lia Almeida de. Indicadores de sustentabilidade para instituições de ensino superior: uma proposta baseada na revisão de literatura. Revista de Gestão Ambiental e Sustentabilidade, v. 8, n. 1, p. 123-144, 2019.

TAUCHEN, Joel; BRANDLI, Luciana Londero. A gestão ambiental em instituições de ensino superior: modelo para implantação em campus universitário. Gestão \& Produção, v. 13, p. 503-515, 2006.

THOMASHOW, Mitchell. The nine elements of a sustainable campus. Sustainability: The Journal of Record, v. 7, n. 3, p. 174-175, 2014.

UMAR, Tariq. An integrated sustainability-management approach for universities. In: Proceedings of the Institution of Civil Engineers-Engineering Sustainability. Thomas Telford Ltd, 2020. p. 344-355. 
Gestão e Desenvolvimento em Revista V. 7, N. 1, jan-jun/2021, p. 71-81. ISSN online: $2446-8738$

Artigo recebido em: 27/05/2021

Artigo aprovado em: 16/08/2021

VELAZQUEZ, Luis et al. Sustainable university: what can be the matter?. Journal of cleaner production, v. 14, n. 9-11, p. 810-819, 2006. 